\title{
Dissecting Moneyball: Improving Classification Model Interpretability in Baseball Pitch Prediction
}

\author{
Kevin Hickey \\ Fairfield University \\ Fairfield, CT, USA, 06824 \\ Kevin.hickey@student.fair- \\ field.edu
}

\author{
Lina Zhou \\ The University of North \\ Carolina at Charlotte \\ Charlotte, NC, USA, 28223 \\ lzhou8@,uncc.edu
}

\author{
Jie Tao \\ Fairfield University \\ Fairfield, CT, USA, 06824 \\ jtao@fairfield.edu
}

\begin{abstract}
Data science, where technical expertise meets domain knowledge, is collaborative by nature. Complex machine learning models have achieved human-level performance in many areas, yet they face adoption challenges in practice due to limited interpretability of model outputs, particularly for users who lack specialized technical knowledge. One key question is how to unpack complex classification models by enhancing their interpretability to facilitate collaboration in data science research and application. In this study, we extend two state-of-the-art methods for drawing finegrained explanations from the results of classification models. The main extensions include aggregating explanations from individual instances to a user-defined aggregation level, and providing explanations with the original features rather than engineered representations. We use the prediction of baseball pitch outcome as a case to evaluate our extended methods. The experiment results of the methods with real sensor data demonstrate their improved interpretability while preserving superior prediction performance.
\end{abstract}

\section{Introduction}

Data science is essentially a confluence of technical and human forms of work. Real-world data science teams are collaborative yet heterogeneous by nature [1]. Central to the collaborative work is trust. Despite the fact that data scientists may have the necessary expertise to juggle between technical skills and domain knowledge, the complex nature of state-of-the-art models makes it hard for data scientists to interpret analytical results, or provide a clear explanation that is applicable to respective domains. As a result, these complex models are faced with trust and acceptance challenges from end-users who likely lack specialized data science knowledge.

As part of the efforts in increasing human trust and acceptance of complex models, the interpretability of classification results has been gaining increasing ground in both research and practices, particularly in areas that hold high stakes, such as the finance and medicine. The expectations for interpretability of classification results is heightened when the users require explicit and actionable explanations in order to accept models, when regulations require unambiguous explanations or rationales behind the decisions, and when the insights are used to support new scientific theory and hypothesis developments [2]. However, research of classification models has predominantly focused on improving model performances (i.e. accuracy). This has motivated the development of complex models such as neural networks and ensemble models, which have achieved near humanlevel performances in some applications. However, these models are generally considered as black-boxes that are hard to interpret by humans. They manifest the traditional accuracy-interpretability trade-off in developing classification models. In other words, models that lead to relatively high performance are more difficult to interpret. Therefore, one key question is whether we can improve model interpretability without negatively affecting its performance. In this research, we aim to answer the research question of unwrapping algorithmic black-boxes by using baseball strike prediction as a case study.

Significant progresses have been made in increasing the interpretability of complex classification models. One common approach is to provide not only classification results but also some of the logic of this classification [3]. Specifically, researchers have looked into the metrics for model interpretability such as model complexity, and the extraction of comprehensible classification results (e.g. rule sets or decision trees) from the black-box models or from the complex models produced by ensembles of classifiers [2]. On the other hand, there are counterarguments that simpler models are not necessarily more interpretable mainly because they contain fewer informative attributes for users to make sense of. Thus, compared with complex models, the monotonicity 
constraints provided by the user or experts in the application domain are better metrics of model interpretability [2].

In this study, we extend the state-of-the-art methods for interpreting classification models, namely Local Interpretable Model-agnostic Explanations (LIME) and SHapley Additive exPlanations (SHAP), by introducing new analytical capabilities to enhance their interpretability for addressing a real-world problem. Major extensions include aggregating explanations from the level of individual instances to a user-defined aggregation level to better support decision making, and providing explanations with the original features rather than their derived complex representations (e.g. engineered features). We design an experiment using sensor data to predict baseball pitch outcomes. The experiment results demonstrate the improved interpretability and sustained superior prediction performances of complex models.

\section{Model Interpretability}

In this section, we discuss the complexity-interpretability trade-off and the motivations for improving model interpretability, and review state-of-the-art methods for model interpretability.

\subsection{Complexity-Interpretability Trade-off}

With increasing rich datasets being collected for analysis in various domains, extracting (implicit) patterns from such datasets becomes more difficult. Traditional machine learning techniques (e.g. regression models, decision trees) are no longer sufficient for such tasks. More advanced modeling techniques (e.g. random forest and deep neural networks [4]) are developed to extract complex patterns (e.g. non-linear patterns) from the datasets. Although more complex models have been trained for better performances with complex datasets, increased model complexity has led to the issue of a decrease in model interpretability. It is believed that some traditional machine learning models yield predictive results with better explanatory power. For instance, in regression models, the coefficients can be used to interpret the effects of input features; and rules can be generated from decision trees to support decision making. Nevertheless, when bagging simple decision trees into random forests, or boosting them with extreme gradient boosting (XGBoost), the interpretability superiority gets lost.

The declined model interpretability has led to several issues. First, the ignorance of why certain decision is made diminishes the decision support role of machine learning models. Real-world data science projects require collaboration and communication among people with diverse domain expertise, who may range from business managers through machine learning specialists/engineers, social scientists, to end users. In addition, the role of data scientists is reflected in their orientation toward solving business and societal problems using machine learning techniques. The interdisciplinary nature of data science research and practice naturally demands the methods to facilitate user interactions with the models (i.e. classifiers) as well as their results [5]. Second, limited model interpretability, often termed as black-box models, faces the challenges of gaining trust and acceptance from users. Users (i.e. analysts) trust models/results that explanations can be drawn from, sometimes regardless of their predictive performances [6]. Third, lack of interpretability goes against the principle of ease-of-use - an important success factor of any system design. One of the key indicators of ease-ofuse in machine learning models is the interpretability of predictive results. Thus, increased model interpretability is crucial for the successful design of any machine learning models [5].

\subsection{Model Agnostic Interpretability}

In view of the diverse representations of various classification models, there are model level interpretability and more generic interpretability issues. One approach to enhancing machine learning model interpretability is to treat original models as black-boxes and draw post hoc explanations from the analytical results by learning explanation models (models used to provide interpretability to the complex counterparts) based on the model results; for instance, we can perturb inputs features to investigate how the black-box model behaves - this approach is termed as model agnostic [7]. One scenario is that human analysts have difficulty in comprehending a model globally if the model structure is very complex. For instance, a large vocabulary size of the text data (i.e. high dimensionality of input data) used to train a sentiment classifier may lead to a complex model. Thus, it is desired to separate the model from its interpretability, in order to preserve its flexibility [8]. Another scenario is that analysts may focus on either the positive (which feature/instances contributes most) or the negative (which feature/instances contributes least) evidences in the prediction. In other cases, analysts need finer-grained information with respect to how different feature values may lead to different model behaviors.

Model interpretability may appear in different forms (e.g. linear models, rules, graphs), and an approach should, accordingly, support as many forms as possible [7]. Regardless of the specific explanation form, an explanation (model) should possess fidelity to the blackbox model - meaning that the prediction from the explanation model should be (to some extent) consistent with 
the original complex model. Since it is very difficult to obtain global fidelity (the explanation model can mimic all the behaviors of a black-box model across the dataset), researchers have instead aimed at achieving local fidelity (behaviors from the explanatory models and the black-box models are consistent at some of the instances). Additionally, it is increasingly recognized that using evaluation metrics (or similarly, loss functions) as the only criteria to compare models, in contrast to incorporating interpretability metrics, has limited applicability [9]. One approach that tackles model agnostic interpretability is LIME [7]. This approach possesses several interesting characteristics, including interpretable data representation, fidelity-interpretability trade-off, sampling for local exploration, and sparse linear explanations.

\subsection{Instance-wise Feature Importance Scoring}

As discussed in Section 2.1, machine learning models generally compromise interpretability with emphasis on performances enabled by increased complexity. As a result, feature ranking (and selection) has become one of the most important tasks in machine learning [10]. Features are ranked by their importance to the predictions (known as feature importance scoring). Feature importance scoring methods can be roughly categorized into two groups, namely global and instance-wise feature importance scoring. Global feature importance scoring measures feature contributions to the prediction based on the overall dataset, which is often used for feature selection purposes. In contrast, instance-wise feature importance scoring measures the relative importance of all input features for a given instance in the dataset. The scoring method often yield a vector of importance scores, associated with all the features [11]. Although the best explanation of a model, or its results, is the model itself, the original models in case of in complex models are deemed not as useful as the best explanations due to their lower interpretability. Thus, a simpler surrogate model that approximates the original model needs to be defined (e.g. a surrogate decision tree model for the original random forest model).

In complex models, where the feature space is often too complex to learn, it is impossible to define the relationship between features and the predictive results. Advanced approaches (i.e. Monte Carlo approximation [9] or nanograms [12]) have been used to search for the optimal values defining such relationships. Recently, cooperative game theory has been used in the context of instance-wise feature importance scoring, in which each feature serves as a player in the game. The latest development in this category is SHAP, which aims to assign importance scores to individual features for each prediction/instance [13]. Compared to previous instance-wise feature importance scoring methods, SHAP possesses unique properties such as local accuracy (the explanation model matches the original model for a given instance), missingness (if a feature has no impact in the original model, it should not have any impact in the explanation model), and consistency (feature contributions should be consistent in both of the original and explanation models).

\section{Methods and Results}

To evaluate the proposed methods for improving model interpretability, we design an experiment to predict the output of baseball pitches (i.e. ball or strike).

\subsection{Proposed Methods}

In real-world decision making processes, investigating the impact of individual features may not be as useful - due to the complexity of the decision problem, it might be more interesting and useful to examine the interactions between features and their combined impacts on the predictions. Users of classification models may prefer explanations of results at a finer granularity rather than at a global level to better inform the course of action. In case of baseball, practitioners may want explanations for individual instances (e.g. pitches). While collaborating with domain experts in collecting requirements for meaningful explanations, they suggest that drawing explanations from individual features may not be very useful but it is necessary to investigate the interaction(s) among different features. To this end, we extend LIME [7] and SHAP [13] in two important aspects:

- The original LIME and SHAP provide instancewise explanations. In view of the size of modern datasets and explanation generality, it can be more efficient and valuable to group similar instances and provide explanations at a group level (e.g. pitches from certain pitcher, a specific type of pitch).

- Although including engineered features (e.g. position_x, position_z) may improve prediction performance, they would not be useful to the end users because those features cannot be directly measured in the field. Thus, we extend LIME and SHAP by drawing explanations from the original rather than the engineered features.

Despite two illustrations that demonstrat the application of LIME [7], there are a few extensions deemed necessary for LIME. For instance, in terms of interpretable data representation, the explanation should be able to penetrate any representation of the features. If there is any engineered features used in the black-box model, the explanation should be reflected upon the original 
features, so that practitioners can better use these explanations in their practices. For instance, in the context of text analytics, if the text is represented in word embedding models, the explanations should be provided on the original words instead of the word embeddings. In addition, linear explanation may be inadequate or not actionable in some contexts. Other forms of explanations, such as decision rules, may become more useful in these scenarios. Additionally, in addition to the instance-wise feature importance scoring, it is important to generalize the interpretation from individual instances, possibly to a group of instances. This can help achieve consistency across similar instances.

\subsection{Analytical Problem and Data}

We use the prediction of the baseball pitch outcome as a case study to evaluate the performance and interpretability of the proposed methods. A pitch is the pitcher throwing a baseball toward the home plate. A called strike (strike) is when a pitch is in the strike zone, or the hitter swings the bat at the pitch and misses; while a called ball ( ball) is a pitch outside of the strike zone and the hitter does not swing ${ }^{1}$. Predicting the outcome of a pitch can help baseball coaches, players, teams, and analysts in identifying areas of improvement or develop counter-strategies. In essence, the prediction of baseball pitch is a binary classification problem. Previous studies have analyzed whether historical pitches would indicate the type of a future pitch [14], [15].

We programmatically collect the data from the StatCast system $^{2}$, which is an analytical platform that uses arrays and sensors placed in all 30 Major League Baseball (MLB) stadiums to track and quantify measurements regarding various aspects of a baseball game, including pitching measurements, hitting measurements, and so forth. We randomly select the pitch data of 350 pitches from the top 400 pitchers (in number of pitches) in the 2018 season of the MLB league (a total of 140,000 pitches). Since sensory data is difficult to impute, we exclude instances that contain any missing value. Finally, our dataset consists of 126,486 instances.

We collect 18 sensory features, along with the name of the pitcher, and the outcome of the pitch. Given that our goal is to predict the outcome of the current pitch, we filtered the features based on their relevance and availability. In addition, we removed 4 features that are strongly correlated with other features, and engineered 2 new features from those 4 features. The following is a final list of input features and prediction target.

\footnotetext{
${ }^{1}$ https://www.umpirebible.com/files/Osborne02.pdf
}

- position_z: a pitch's ending vertical position calculated using the vertical release position and the vertical movement from the catcher's perspective;

- position_x: a pitch's ending horizontal positioncalculated using the horizontal release position and the horizontal movement from the catcher's perspective;

- p_throws: hand with which the pitch is thrown (left/right);

- vx0: horizontal velocity (ft/sec) of the pitch, determined at $y=50$ feet;

- vy0: velocity $(\mathrm{ft} / \mathrm{sec})$ of the pitch in the direction toward the catcher, determined at $y=50$ feet;

- $\quad \mathbf{z z 0}$ : vertical velocity $(\mathrm{ft} / \mathrm{sec})$ of the pitch, determined at $y=50$ feet;

- release_spin_rate: spin rate of the pitch;

- $\quad$ pitch_name: pitch type such as four-seam fastball and curve ball.

- $\quad$ sz_top: top of the strike zone when the ball is halfway to the plate;

- $\quad$ Sz_bot: bottom of the strike zone when the ball is halfway to the plate;

- $\quad$ player_name: the name of the pitcher;

- outcome: resulting pitch $(1=$ strike, $0=$ ball $))$.

The data is imbalanced, with a distribution ratio of roughly 1:2 between strike and ball. Thus, we performed random under-sampling on the ball pitches. The results of correlation analysis show that the selected feature are strongly correlated with the target variable.

\subsection{Classification Models and Results}

We implemented two categories of classification techniques in this study: traditional (baseline) models such as logistic regression (LR) and decision tree (DT), and complex models such as random forest (RF) and XGBoost $(\mathrm{XG})$. Decision trees, classification rules or decision tables tend to be higher in interpretability than mathematical equations (cf. [2]), and thus we choose DT as one of the baseline method in this study. On the other hand, the complex models are capable of capturing complex (e.g. non-linear) patterns in the data at the cost of interpretability. The data did not go through traditional preprocessing steps (e.g. scaling, skewness handling) except for one-hot encoding on the categorical features (pitch_name, $p \_$throws) due to concerns about the possibly negative impacts of those steps on the interpretability of the prediction results.

2 www.baseballsavant.com 
All the models included in this study were evaluated with a 5-fold cross-validation. In addition, the random search method was employed to search for the optimal hyperparameters in all the models. The evaluation metrics include binary cross-entropy accuracy (ACC), Area Under Receiver Operating Characteristics Curve (AUC), and F1-score. AUC values range from 0 to 1 [16]. It represents random guessing when $\mathrm{AUC}=0.5$; and perfect prediction while $\mathrm{AUC}=1$. F1-score is a harmonic mean of precision and recall. To validate the results interpretations derived from the models, we consulted domain experts.

The predictive results of all models are reported in Table 1. The highest value for each evaluation metric is marked in bold face. The result show that the advanced modeling techniques (RF, XG) outperforms traditional machine learning techniques (LR, DT) in the predictive power. Particularly, the XGBoost model (XG) outperforms all other models in accuracy (.915), AUC (.937), and F1-score (.884).

\section{Table 1. Predictive Results of Models}

\begin{tabular}{l|lll} 
MODEL & ACC & AUC & F1 \\
\hline LR & .574 & .591 & .492 \\
DT & .439 & .575 & .523 \\
RF & .830 & .824 & .754 \\
XG & $\mathbf{. 9 1 5}$ & $\mathbf{. 9 3 7}$ & $\mathbf{. 8 8 4}$
\end{tabular}

\subsection{Results Interpretation of Baseline Methods}

As discussed in Section 2.1, baseline mdoels (LR \& DT) possess better interpretability in the predictive results. For instance, in the LR model, the signages of the coefficients in the trained model indicate whether a feature positively or negatively impacts the prediction. We report the signages of coefficients in the trained LR model (see Table 2). In Table 2, the top-3 features that are positively correlated with the strike are: $s z$ top (.64), pitch_Sinker (+.53), and pitch_2-seam_fastball (+.41), which indicate that the top of the strike zone, as well as two variants of the fast ball pitches (Sinker and 2-Seam Fastball) would increase the likelihood of throwing a strike. On the contrary, the top-3 features that are negatively correlated with the strike are: $s z$ bot $(-1.2)$, pitch_Split_Finger (-.63), and pitch_ChangeUp (-.49), increase the likelihood of throwing a ball. The domain experts verified the above explanations. They confirm that pitchers typically have a better control of a fastball or its variants, rather than an off-speed pitch (i.e. Change-up, Split-Finger); thus, the latter decreases the likelihood of a strike. Additionally, the results also suggest that the thresholds of the strike zone also contribute to the likelihood of throwing a strike: if the top of the strike zone (sz top) is higher, and the bottom of the strike zone (sz_bot) is lower (the batter has a larger zone), it reduces the chance of a pitch resulting outside of the zone (which is automatically a ball).

\begin{tabular}{l|l}
$\begin{array}{l}\text { Table 2. Coefficients in the Trained LR Model } \\
\text { FEATURE }\end{array}$ & COEFFICIENTS \\
\hline position_x & -.04 \\
position_z & +.27 \\
release_spin_rate & +.00 \\
sz_top & +.64 \\
Sz_bot & -1.2 \\
p_throws_l & -.06 \\
p_throws_r & +.08 \\
vx0 & -.02 \\
vy0 & +.02 \\
vz0 & +.08 \\
pitch_2-seam_fastball & +.41 \\
pitch_4-seam_fastball & +.35 \\
pitch_changeup & -.49 \\
pitch_curveball & -.10 \\
pitch_cutter & +.06 \\
pitch_sinker & +.53 \\
pitch_slider & -.10 \\
pitch_split_finger & -.63
\end{tabular}

The results suggest that the result explanations from the traditional models (i.e. LR and DT) have high predictive power. Despite that the advanced, high performance models and the traditional, high interpretability models share common explanations, there are some noticeable differences between their explanations. For instance, the feature release_spin_rate is ranked slightly higher in the advanced than the traditional models, but none of the pitch types received high rankings in LR \& DT models. Under these circumstances, it is difficult for practitioners (e.g. analysts) to determine which group of models to trust in their practices.

The explanations for Decision Tree (DT) results can come in two forms: decision rules and feature importance scoring. In order to maintain the readability of the tree structure, we limit the depth of the tree to 4 . Based on the domain experts' review of the decision rules generated by DT, and the feature importance scoring, we selected a few informative rules. Two sample rules are listed in the following:

- If a pitch is not moving downward at $8.955 \mathrm{ft} / \mathrm{sec}$ and is not a type of Change-up, it is a strike;

- If the lateral movement of a pitch is below 9.23 $\mathrm{ft} / \mathrm{sec}$ to the right in the catcher's perspective, it is a strike.

Compared with other models, the DT model performed better in predicting ball (the negative class) than strike (the positive class). In contrast, the RF and XG models are more accurate in predicting the target variable. Since they are both decision tree 
based approaches, so we extract their feature importance scoring from them separately (see Figure $1)$.

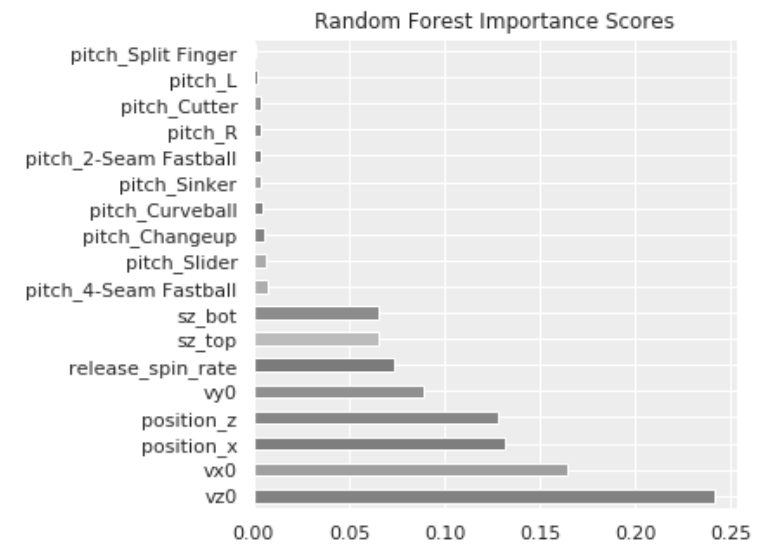

(a) RF Model

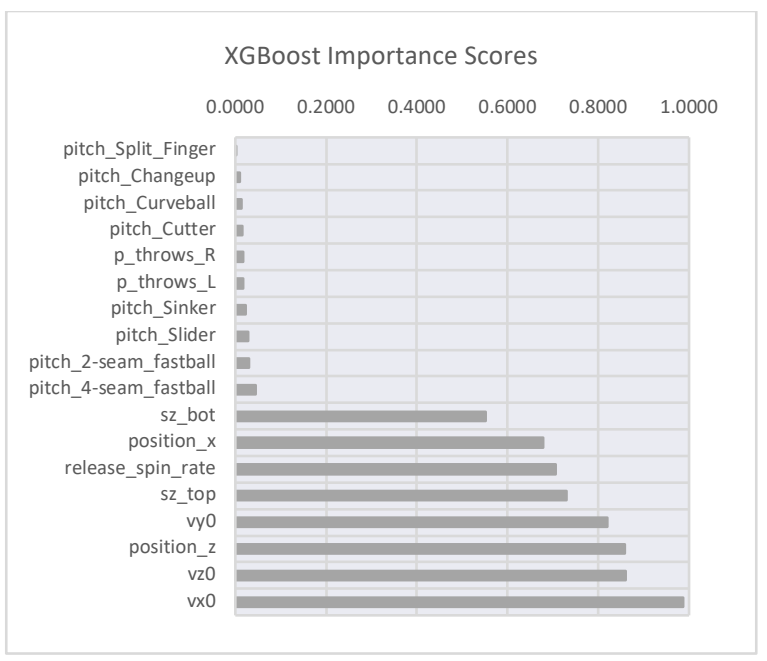

(b) XG Model

Figure 1. Feature Importance Scoring

We make some interesting observations from the figure. Firstly, the vertical speed of the pitch (vz0) remains is one of the top features in the prediction, and the horizontal speed (vx0) is ranked among the top by both RF and XG. Secondly, consistent with the LR and DT models, the final positions of the pitch (position_ $x$ and position_z) are ranked high by both of the complex models. Similar to the results of LR, the measurements of the strike zone ( $s z$ top and $s z$ bot) are selected as important features by both RF and $\bar{X}$ G models.

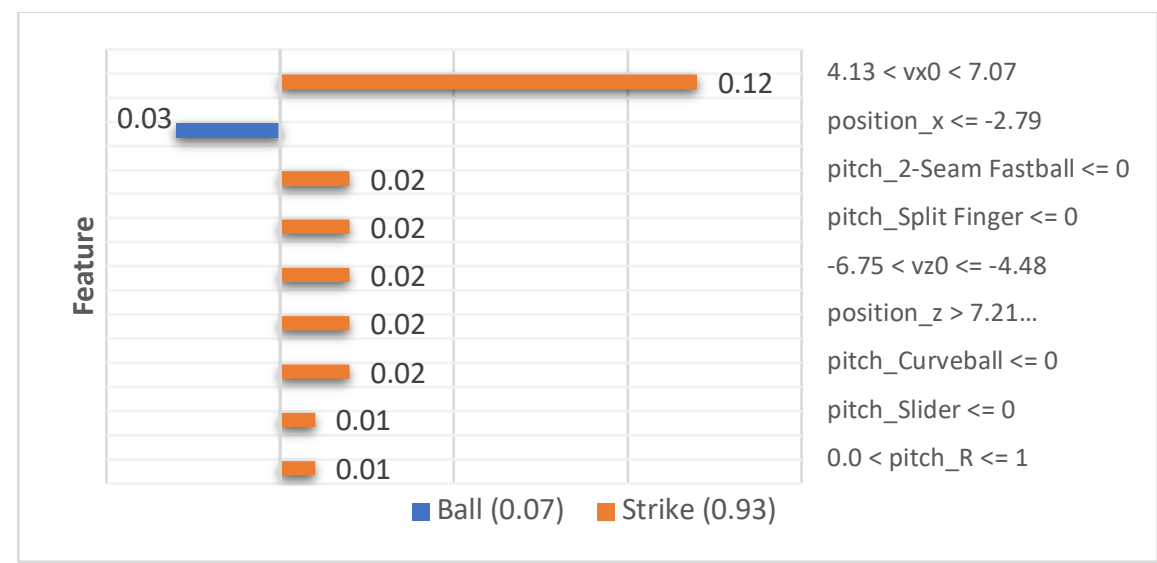

Figure 2. LIME Results of an Individual Pitch

\subsection{Results Interpretation of Our Extended Methods}

We report the results of our extended LIME method from our best performing XGBoost model for individual pitches in Figure 2. For the sake of space, we randomly select one instance from the top ranked predictions (in terms of accuracy) and interpret its results. Figure 2 shows that the XG model is $93 \%$ confident that the specific pitch is a strike; and the top contributing feature to this prediction (right part of the figure) include the releasing lateral speed $(v x 0)$, ending horizontal position (position_x), and whether this pitch a Curve Ball is not. We can draw the following sample decision rules from the figure: 
- If a right-handed pitcher throws a pitch that is not a 2-seam fastball, a curveball, or a slider, the pitcher controls the lateral speed (at 50 feet from the releasing point) in the range of between 7.07 $\mathrm{ft} / \mathrm{sec}$ and $4.13 \mathrm{ft} / \mathrm{sec}$ to the right, and the vertical speed is between 4.48 and $6.75 \mathrm{ft} / \mathrm{sec}$ downwards, then it increases the likelihood of the pitching ending up being a strike.
- If the ending horizontal position is more than 2.79 $f t$ to the left (against the center of the strike zone), then it decreases the likelihood of the pitching ending up being a strike.

Actual Value: 1

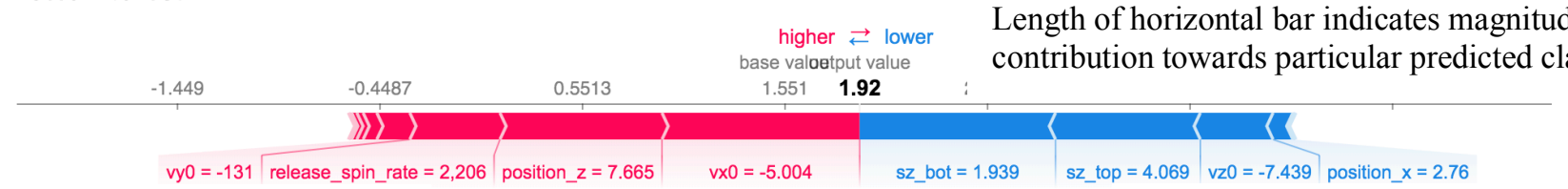

Features in red contribute to prediction of strike.

Features in blue contribute to prediction of ball.

Subsequently, we reverse-engineered the feature position_ $x$ : if the release position is $3 \mathrm{ft}$. to the left of the pitcher, then the lateral movement between the release and the home plate should be less than $0.21 \mathrm{ft}$.

We report the results of the extended SHAP method for individual pitches from our best performing $X G$ model in Figure 3. In the figure, for a particular pitch resulting in strike, features shown in blue contribute negatively to the prediction, where the magnitudes of the impact is expressed in the length of the bar, meaning the particular values of features such as ending horizontal position (position_x), vertical speed $(\mathrm{vz} 0)$, and strike zone top ( $s z$ top) and bottom ( $s z$ bot) decrease the likelihood of the pitch being a strike. Thus, the SHAP results can be used to assess the global (dataset level) feature importance scoring. The more instance-wise feature importance scoring is aligned with the global feature importance scoring, the more consistent and reliable is the model.

We also analyzed the pairwise feature interaction, and their effects on prediction results. For example, the effects of interaction between the vertical speed $(\mathrm{vz} 0)$ and a specific type of pitch (Slider) are plotted in Figure 4. A slider is a particular type of pitch that breaks downward along the trajectory. For instance, if the speed of the pitch moving downward is faster than $5 \mathrm{ft} / \mathrm{sec}$ at the point of $50 \mathrm{ft}$. away from the catcher on the pitch trajectory, there is a higher likelihood that the pitch results in a ball. Additionally, if the vertical speed $(v z 0)$ lies in the range between both $2.5 \mathrm{ft} / \mathrm{sec}$ downwards and $2.5 \mathrm{ft} / \mathrm{sec}$ upwards, the likelihood of a pitch resulting in as a strike increases (peaking at approximately $0 \mathrm{ft} / \mathrm{sec}$ ). This observation makes sense even with the basic knowledge

${ }^{3}$ Due to the computational complexity, we randomly sample 5,000 pitches from the analysis data. about baseball: for a Slider pitch, the vertical speed should be in a certain range so that the pitch can land in the strike zone.

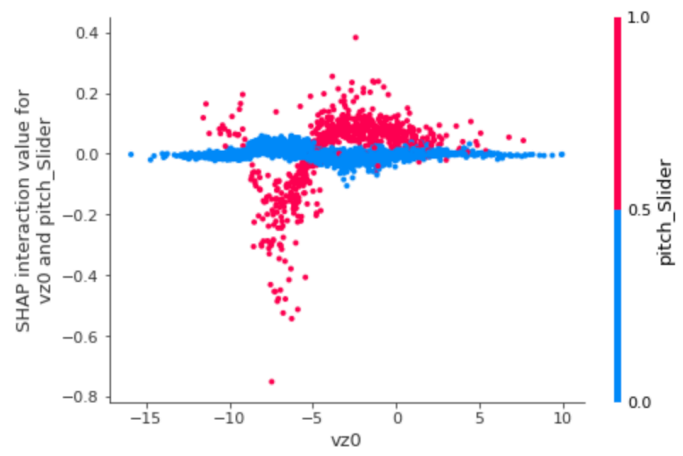

Figure 4. Pairwise Feature Interaction Analysis ${ }^{3}$

We can also observe from Figure 4 that the interactions between these two features are non-linear. The feature importance scoring from the XG model after considering the interactions between features is shown in Figure 5. A comparison between Figure 5 and Figure 1 (c) shows that, regardless of feature interaction, some features (e.g. $v z 0, v x 0$ ) consistently demonstrate their importance for making predictions. Figure 5 also shows how an individual feature, in combination of all other features, impacts the prediction. For instance, the lower the vertical speed $(v z O)$ is, the lower likelihood a pitch results in a strike. The observations provide evidence for increased generalizability in terms of explanations of high quality predictive results. 
To demonstrate our extension of SHAP to a user-defined aggregation level, we select a specific pitcher (pseudo name: AN), and then analyze 3 randomly selected fastball pitches of 2 types each (2 4-Seam Fastballs and 22-Seam Fastballs), . Our method is able to capture some subtle differences between the 2 fastball types (see Figure 6). Specifically, the XG model is highly confident $(>.85)$ about the first 2 predictions. The first prediction is a ball, and the combination of the release vertical position (position_z<=5.91) and the horizontal speed $(v x 0>7.07)$ are strong indicators of the prediction. The next two predictions are strikes: compared to the first prediction, the main difference lies in the vertical $(v z 0)$ and horizontal $(v x O)$ speed. Because of the high confidence in these predictions, the thresholds of $\{v z 0:-2.03 \mathrm{ft} / \mathrm{sec} ; v x 0: 7.07 \mathrm{ft} / \mathrm{sec}\}$ can be used as a guide in pitcher training/coaching. Additionally, we select a misclassified prediction in which the model learns contradicting patterns from the top-3 important features: the top feature $(v z 0)$ shows a strong impact on predicting a strike; whereas the next two top features show strong indications toward prediction a ball. This finding confirms that complex machine learning models (e.g. XGBoost) are able to learn patterns from feature interactions. Furthermore, we observe that between prediction 2 and prediction 3 , the values of $v x 0$ lie on both sides of the threshold, which provides evidence that the $\mathrm{XG}$ model is capable of capturing the differences between different types of fastballs.

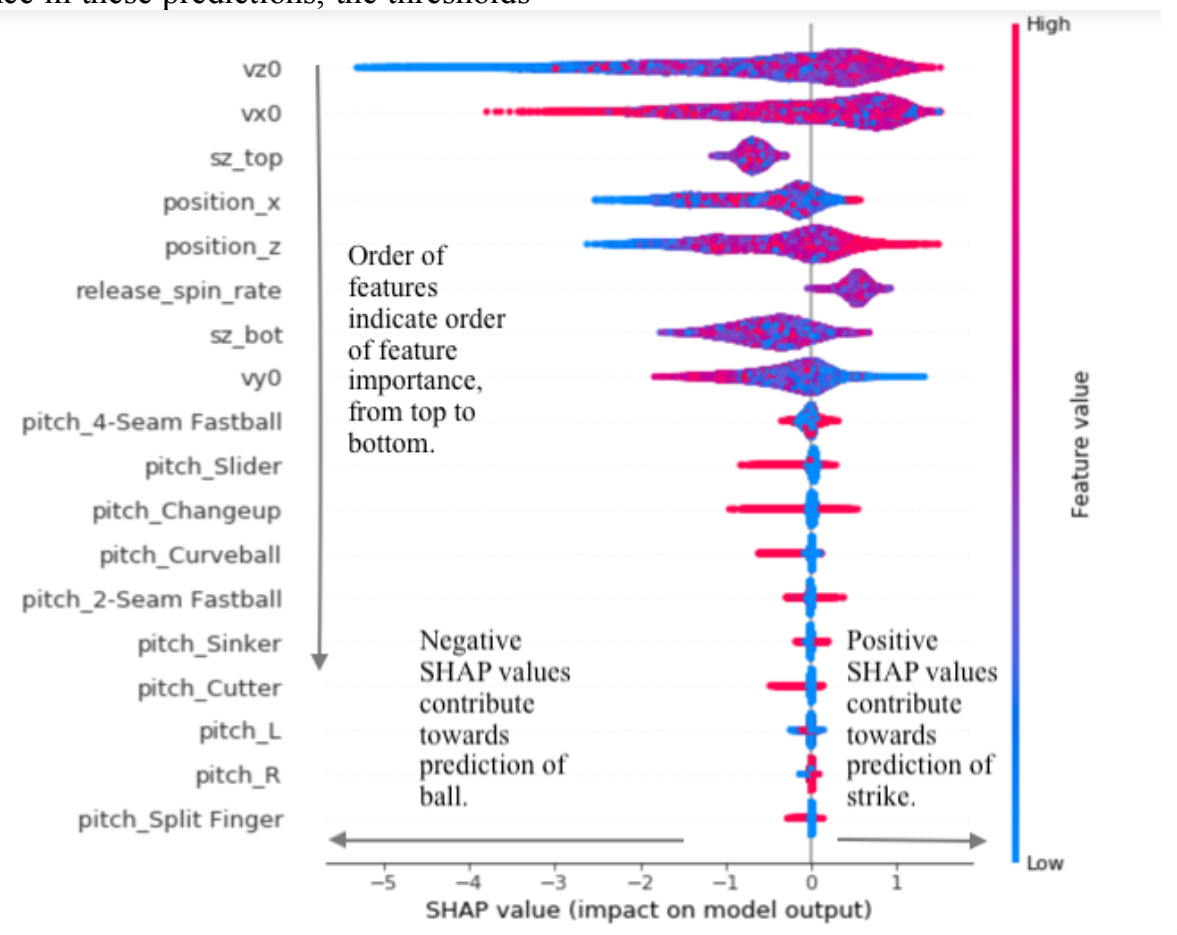

Figure 5. Importance Scoring for Feature Interaction based on XG Model Results

\section{Discussions}

Although we can directly draw explanations (in different forms) from the predictive results of the LR and DT models, their performances are inferior to complex models (i.e. RF, XG) in terms of the predictive power. As a result, users or analysts may not accept the results from traditional baseline models (i.e. LR, DT) due to their poor performances. Complex models like RF and $\mathrm{XG}$ are able to achieve better performance at the cost of interpretability. In addition, the interpretability of the traditional models (e.g. LR, DT) above are limited to the global level. In other words, the influences of features on the model results are interpretable from a general, holistic perspective of all instances of a dataset, but are not interpretable on specific, individual instances. Furthermore, we present interpretation of results in decision rules, which are actionable to support domain-specific decisions.

This research contributes to the data science research and practice in multiple aspects. Firstly, it provides new evidences that interpretability methods, specifically LIME and SHAP, can help unpack complex, black-box models. Secondly, it extends the above methods by introducing new analytical capabilities to enhance their interpretability for addressing a real-world problem. The main extensions include aggregating explanations from the level of individual instance to a user-defined group 
level to better support decision making and providing explanations to the original features rather than their derived complex representations (e.g. engineered features). Thirdly, this study represents the first effort in predicting the outcome of the current pitch by employing sensory data from stadiums. While investigating the interpretability of prediction results, we not only discovered finer-grained representations of interpretations, but also validated the explanations with domain experts. The explanations drawn from the results of the current study can be directly used to inform baseball coaching and training, which in turn augment human trust in the prediction results of complex models.

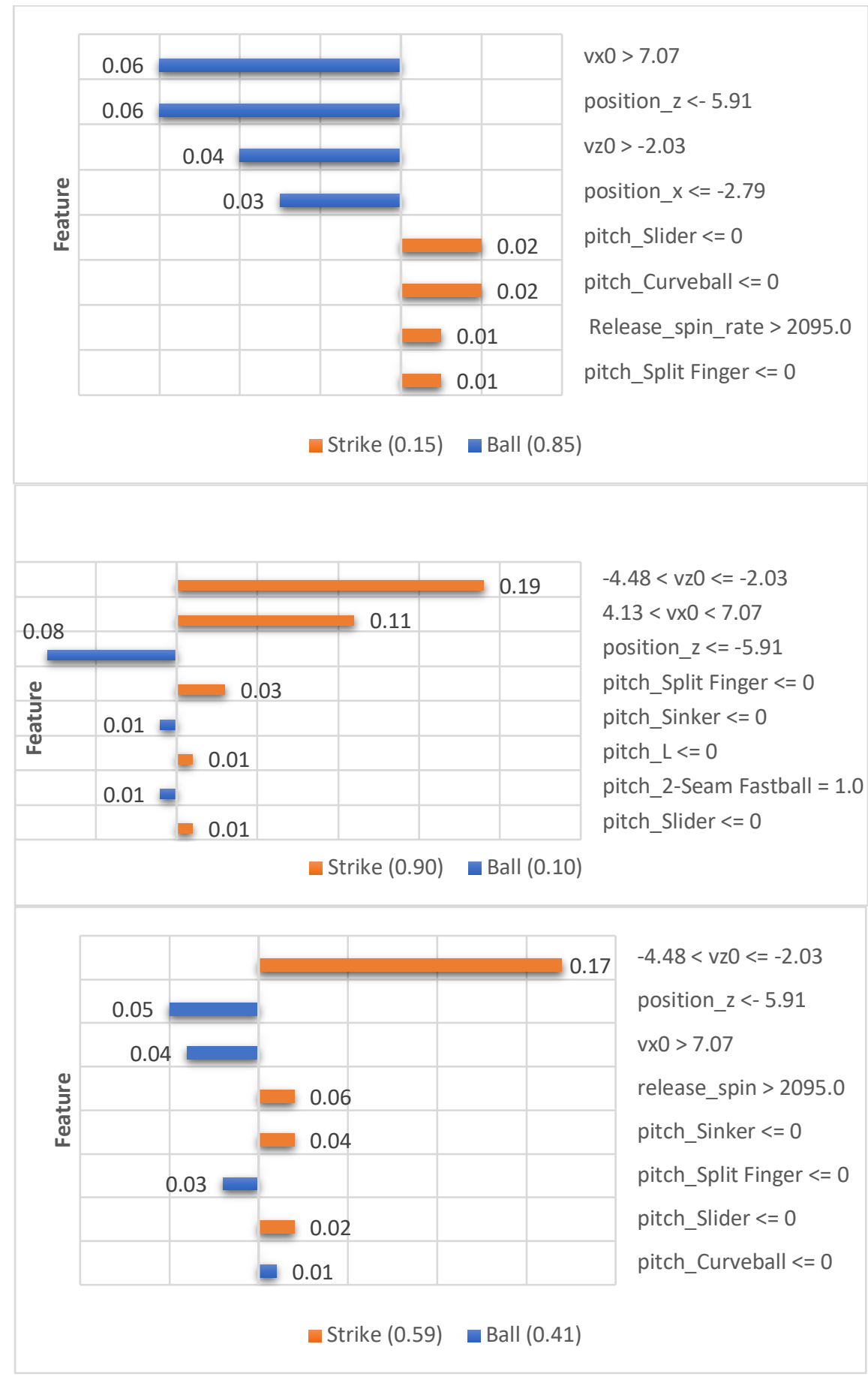

Figure 6. Aggregated LIME Interpretation at Pitcher Level 
We also learned a few lessons from applying LIME and SHAP on the predictive results, which offers recommendations for future analyses. Firstly, in addition to interpretability methods, LIME/SHAP can also be used to support prescriptive and diagnostic analyses. For the prescriptive purposes, LIME/SHAP provide the means for the analysts to gauge how models behave. In other words, LIME/SHAP show light on not only (complex) patterns embedded in the data, but also how a model makes predictions (i.e. what combination/interaction of features informed the prediction). This is particularly useful for designing new algorithms, or tuning a trained model for better performances. For diagnostic purposes, LIME/SHAP can help analysts identify condition(s) that might lead to misclassifications. Secondly, speaking from our own experiences, SHAP is computationally more expensive when compared with LIME; thus, it is deemed necessary to develop new approaches to optimizing the search for optimal SHAP values for the features (e.g. [11]) . Last but not the least, in the context of the baseball pitch prediction, SHAP appears to be superior to LIME in terms of interpretability. For instance, SHAP provides support at both instance and the overall dataset level, and provides support for understanding the interaction(s) between a specific pair of features.

\section{Conclusion}

As machine learning models grow more complex, effective communication of the (prediction) results of these models is being recognized as one of the most important factors in gaining trust from analysts/end users in the data science field. In this study, we propose extensions to the state-of-the-art interpretability methods, namely LIME and SHAP, by equipping them with the abilities to provide explanations on the original features, and aggregate explanations at the user-defined decision levels. The results of our empirical evaluations with the prediction of baseball pitch outcomes demonstrate enhanced interpretability of the extended approaches.

\section{References}

[1] S. Passi and S. J. Jackson, "Trust in Data Science: Collaboration, Translation, and Accountability in Corporate Data Science Projects," in In Proceedings of the ACM on Human-Computer Interaction, 2018, vol. 2, no. CSCW, Article 136, pp. 136:2-136:28.

[2] A. A. Freitas, "Comprehensible Classification Models a position paper," SigKDD Explorations, vol. 15, no. 1, pp. $1-10,2014$
[3] J. Burrell, "How the Machine 'Thinks:' Understanding Opacity in Machine Learning Algorithms," Big Data \& Society, no. Janurary-June, pp. 1-12, 2016.

[4] C. Krauss, X. Anh, and N. Huck, "Deep neural networks , gradient-boosted trees, random forests : Statistical arbitrage on the S\&P 500," European Journal of Operational Research, vol. 259, no. 2, pp. 689-702, 2017.

[5] L. Zhou, S. Pan, J. Wang, and A. V. Vasilakos, "Machine learning on big data: Opportunities and challenges," Neurocomputing, vol. 237, no. December 2016, pp. 350-361, 2017.

[6] R. Piltaver, M. Luštrek, M. Gams, and S. Martinčić-Ipšić, "What makes classification trees comprehensible?," Expert Systems with Applications, vol. 62, pp. 333-346, 2016.

[7] M. T. Ribeiro, S. Singh, and C. Guestrin, "Why Should I Trust You?' Explaining the Predictions of Any Classifier," in Proceedings of the 22nd ACM SIGKDD International Conference on Knowledge Discovery and Data Mining KDD '16, 2016, pp. 1135-1144.

[8] G. Shmueli and O. R. Koppius, "Predictive Analytics in Information Systems in Research," MIS Quarterly, vol. 35, no. 3, pp. 553-572, 2011.

[9] E. Strumbelj and I. Kononenko, "An Efficient Explanation of Individual Classifications using Game Theory," Journal of Machine Learning Research, vol. 11, pp. 1-18, 2010.

[10] A. Razmjoo, P. Xanthopoulos, and Q. P. Zheng, "Online feature importance ranking based on sensitivity analysis," Expert Systems with Applications, vol. 85, pp. 397-406, 2017.

[11] J. Chen, L. Song, M. J. Wainwright, and M. I. Jordan, "L-Shapley and C-Shapley: Efficient Model Interpretation for Structured Data," 2018.

[12] E. Štrumbelj and I. Kononenko, "Explaining the Predictions of an Arbitrary Prediction Model: Feature Contributions and Quasi-nomograms," in Human and Machine Learning: Visible, Explainable, Trustworthy and Transparent, J. Zhou, F. Chen, S. A. Cambo, and D. Gergle, Eds. Cham: Springer International Publishing, 2018, pp. 139157.

[13] S. M. Lundberg and S. I. Lee, "A Unified Approach to Interpreting Model Predictions," Advances in Neural Information Processing Systems, pp. 4765-4774, 2017.

[14] P. Hoang and H. T. Tran, "A Dynamic Feature Selection Based LDA Approach to Baseball Pitch Prediction," in Fast Dependency Parsing using Distributed Word Representations, 2015, p. pp.125-137.

[15] G. Sidle and H. Tran, "Using multi-class classification methods to predict baseball pitch types," Journal of Sports Analytics, vol. 4, no. 1, pp. 85-93, 2017.

[16] M. Wasikowski and X. Chen, "Combating the small sample class imbalance problem using feature selection," IEEE Transactions on Knowledge and Data Engineering, vol. 22, no. 10, pp. 1388-1400, 2010. 